Verified the effectiveness of AsCpf1 in a variety of species

\title{
Verified the effectiveness of AsCpf1 system in a variety of vertebrate species
}

Zhuo $\mathrm{Li}^{1,2,4}$, Zhaoying Shi ${ }^{3,4}$, Nana Fan ${ }^{1,2,4}$, Yongqiang Chen ${ }^{1,2,4}$, Jing Guo ${ }^{1,2}$, Jingchun

$\mathrm{Wu}^{1,2}$, Hong Song ${ }^{1,2}$, Shilong $\mathrm{Chu}^{1,2}$, Kunlun $\mathrm{Mo}^{1,2}$, Bentian Zhao ${ }^{1,2}$, Zhen Ouyang ${ }^{1,2}$,

Dandan $\operatorname{Tian}^{3}$, Shaoyang Zhao ${ }^{1,2}$, Jieying Zhu ${ }^{1,2}$, Jiekai Chen ${ }^{1,2}$, Yonglong Chen ${ }^{1,2,3}$,

Liangxue Lai ${ }^{1,2}$ \& Duanqing Pei ${ }^{1,2}$.

1 Key Laboratory of Regenerative Biology, South China Institute for Stem Cell Biology

and Regenerative Medicine, Guangzhou Institutes of Biomedicine and Health,

Chinese Academy of Sciences, Guangzhou 510530, China.

2 Guangdong Provincial Key Laboratory of Stem Cell and Regenerative Medicine,

South China Institute for Stem Cell Biology and Regenerative Medicine, Guangzhou

Institutes of Biomedicine and Health, Chinese Academy of Sciences, Guangzhou

510530, China.

3 Department of Biology, South University of Science and Technology of China,

Shenzhen, 518055, China.

4 These authors contributed equally to this work. Correspondence should be addressed to Z. L. (li_zhuo@gibh.ac.cn) Phone number: +86 18664604191. 
bioRxiv preprint doi: https://doi.org/10.1101/272716; this version posted February 27,2018 . The copyright holder for this preprint (which was not certified by peer review) is the author/funder, who has granted bioRxiv a license to display the preprint in perpetuity. It is made available under aCC-BY-NC-ND 4.0 International license.

Verified the effectiveness of AsCpf1 in a variety of species

Keywords: gene editing, CRISPR/Cpf1, vertebrate embryos. 
Verified the effectiveness of AsCpf1 in a variety of species

CRISPR/Cpf1 system is a novel genomic editing tool. Because of its more sophisticated components, and lower off-target rate, it has the potential to become a better gene-editing tool. Previous reports showed that CRISPR/Cpf1 could work effectively in multiple species. But our data show that AsCpf1 activity has a big difference in different vertebrates. Using in vitro experiments, we finally learned that the difference between species is due to temperature. 
Verified the effectiveness of AsCpf1 in a variety of species

In recent years, gene-editing tools have been greatly developed, and are widely used.

CRISPR/Cpf1 system is a new member of the gene-editing technique family, and

current researches have shown its better ease of use(Jiang et al., 2017; Kim et al.,

2016b; Port and Bullock, 2016; Tang et al., 2017; Zetsche et al., 2015). This system

can target and cut specific double-stranded DNA (dsDNA) with only two elements:

CRISPR RNA (crRNA) and Cpf1 protein. CRISPR RNA recognizes and targets

specific DNA sequence, and guides Cpf1 protein to assemble a ternary complex;

ultimately, Cpf1 protein cut the targeted dsDNA. Cpf1 protein is smaller than Cas9,

and crRNA, only $43 n t($ including $23 n t$ recognition sequence), is also shorter than

sgRNA. Based on reports, Cpf1 has outstanding performance in the off-target

profile(Kim et al., 2016a; Kleinstiver et al., 2016). These advantages have shown this

system is potentially better than ever before gene-editing tools. From plants to

animals, CRISPR/Cpf1 has been validated in multiple species(Kim et al., 2016b; Port

and Bullock, 2016; Tang et al., 2017; Toth et al., 2016; Zetsche et al., 2015), but some

commonly used laboratory animal models have been reported rarely. Thus, we want

to test the CRISPR/Cpf1 system on embryos of four commonly used model animals: 
Verified the effectiveness of AsCpf1 in a variety of species

mice, porcine (female embryos), frogs(Xenopus), zebrafish; in this study, we have chosen AsCpf1, because of its significant functionality of cutting dsDNA(Kim et al., 2016a; Kim et al., 2016b; Kleinstiver et al., 2016; Zetsche et al., 2015), as testing subject on embryos of these four commonly used models.

In the mouse genome, we chose mTet2 locus as target gene loci, and selected four targets for this locus. Validated CRISPR/Cas9 target in mTet2 was used as positive control(Wang et al., 2013). In order to obtain more reliable results in mouse embryos, we first assessed the effectiveness of selected target sites in mouse embryonic stem cells (mESCs) (Supplementary Figure 1a, b). We transfected PiggyBac plasmids that contain AsCpf1 gene and puromycin resistance gene into mESCs. After puromycin selection, transgenic cells stably expressed AsCpf1 proteins. Then, the crRNAs were transfected into transgenic cells. 48 hours after transfection, cells were collected to do double strand DNA breaks (DSB) assay. The results show all mTet2 targets were cut (Supplementary Figure 1c, d), and the highest cleavage efficiency is crm Tet2-1, 50\% (Supplementary Figure 1c, Supplementary Table 1); sgRNA 
Verified the effectiveness of AsCpf1 in a variety of species

cleavage efficiency at the same gene locus is $95 \%$ (Supplementary Figure 1c, d).

After screening, we chose crm Tet2-1 as target for testing CRISPR/Cpf1 system in mouse embryos.

We optimized the nuclear localization signal (NLS) prior to the mouse experiment

(Supplementary Figure 1e), and found that the addition of NLS at the N-terminus of AsCpf1 did not significantly alter the function of AsCpf1 (Supplementary Figure 1f, g). So in the follow-up experiments we only add NLS to the C-terminus.

Referring CRISPR/Cas9 mouse embryo operating standard(Wang et al., 2013), 50ng/ul AsCpf1 mRNA and 20ng/ul crRNA were used. Injected embryos were cultured to the blastocyst stage, and were collected to prepare genome. After Sanger sequencing, we found that crm Tet2-1 target site has been cut and had indel (efficiency: 32\%) (Fig a, Supplementary Figure 2a, Supplementary Table 2).

In pigs, we have directly operated on parthenogenetic embryos. Four target sites at 
Verified the effectiveness of AsCpf1 in a variety of species

pMSTN locus and two at pSall1 locus have been chosen. Validated CRISPR/Cas9

targets in pMSTN and pSall1 were used as positive control. Referring CRISPR/Cas9

pig parthenogenetic embryo operating standard(Zhou et al., 2015), 200ng/ul Cpf1

mRNA and 20ng/ul crRNA were used. Two target sites of pMSTN have occurred DSB

while their cleavage efficiencies are both lower than 20\% (Fig b, Supplementary

Figure 2b, c, Supplementary Table 3). Another target site at pSall1 locus also has

DSB and it has a little higher cleavage efficiency - 33\% (Fig c, Supplementary

Figure 2d, Supplementary Table 4).

In zebrafish and xenopus, we chose two genes for each specie: zUrod, zTyr for

zebrafish and $x P 48, x$ Tyr for xenopus. In addition, four target sites of each gene were

designed. Validated CRISPR/Cas9 targets were used as positive control(Guo et al.,

2014; Qin et al., 2015). The data of mRNA injected embryos show no significant

occurrence of DSB at these target sites (Supplementary Figure 3a, b, c, d). There is

a suspected difference between control groups and one experimental group

(Supplementary Figure 3a). After Sanger sequencing, those are polymorphism at 
Verified the effectiveness of AsCpf1 in a variety of species

non-target area (data no to show). So, we suspected this result may be because that the number set of targets we detect is too small to be representative of AsCpf1 capacity. So we extended the target sets of these two species, the results show that there is still no DSB present (Supplementary Figure $\mathbf{3 e}, \mathbf{f}, \mathbf{g}, \mathbf{h}$ ).

In order to understand whether the target selection or some other issue is the reason of these negative results. We designed an exogenous target detection(ETD) system: cloned the mouse Tet2 Cpf1 target into the vector that expresses GFP, named pTg-mTet2 (Supplementary Figure 4a), it was co-transfected with AsCpf1 expression plasmid and crRNA into human HEK293T cells. The results showed that exogenous m Tet2 target was edited in human cells (Fig d, Supplementary Figure 4b). After verifying the ETD system can effectively detect the effectiveness of exogenous target, we chose several zebrafish and xenopus targets to test on this system. Surprisingly, it has been found that all those targets are high cleavage efficiency in the HEK293T cells (Fig d, Supplementary Figure 4d). 
Verified the effectiveness of AsCpf1 in a variety of species

Exogenous target detection experiments have shown that the appearance of negative

results is not due to target selection. So we turned our attention to other possible

reasons. The culture temperatures of zebrafish and $\mathrm{x}$ enopus were less than $30^{\circ} \mathrm{C}$,

which is far below the culture temperature of the two mammalian embryos. This

makes us wonder if temperature is the deciding factor - AsCpf1 protein activity was

lacked in the non-optimal working environment. So we did the in vitro enzyme activity

test. A certain unit of purified AsCpf1 protein can be very effective in cutting DNA at 37

${ }^{\circ} \mathrm{C}$ (Supplementary Figure 5a, b). The same goes for reactions that add embryonic

lysates at $37^{\circ} \mathrm{C}$, however, our experimental results showed that the activity of AsCpf1

was significantly lower at $28.5^{\circ} \mathrm{C}$ and $25^{\circ} \mathrm{C}$ (Fig e).

Since injected mRNAs take a few hours to translate into protein in the embryos, and

AsCpf1 protein is greatly reduced in activity at low temperatures, the strategy of

injecting mRNAs is apparently inappropriate. We used crRNAs from those target that

had been detected in the ETD system, mixed with AsCpf1 protein and injected into

xenopus and zebrafish embryos. By T7E1 DSB assay, there was a slight difference 
Verified the effectiveness of AsCpf1 in a variety of species

between the experimental and control groups in each of the two species (Fig $\mathbf{f}, \mathbf{g})$. We

confirmed that there was a single-base mutation in both experimental groups by

sanger sequencing (Fig $\mathbf{h}, \mathbf{i})$.

One finer temperature gradient experiment showed that AsCpf1 protein had the highest activity at about $37{ }^{\circ} \mathrm{C}(\mathrm{Fig} \mathbf{j}$, Supplementary Table 5, 6), while had a significant activity decrease as the temperature increased or decreased (Fig k,

Supplementary Table 7). We believe that temperature may be the reason why AsCpf1 cut only a small amount of DNA in the two low temperature species -temperature sensitivity.

CRISPR/Cpf1 system has significant advantages in working mechanism. Our data

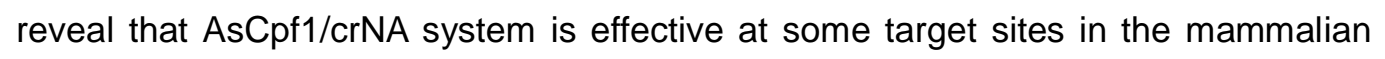
embryos. Unfortunately, it did show only a very low-level function in zebrafish and xenopus, which are two classic developmental biology animal models. This may be due to the sensitivity of $\mathrm{AsCpf} 1$ to temperature. And prolonged the reaction time under 
Verified the effectiveness of AsCpf1 in a variety of species

low temperature conditions is an effective way (Supplementary Figure 5c), which

can also explain why the low temperature species that have been reported can

effectively be genetically modified after obtaining transgenic lines(Port and Bullock,

2016; Tang et al., 2017). But, it is still unknown that whether to extend the reaction

time in non-optimal conditions will increase the risk of off-target? If scientists want to

develop CRISPR/Cpf1 system into a mature gene-editing tool, more test and

optimization on different species are needed. In this study, we only discussed the

effectiveness of the CRISPR/Cpf1 systems in four different animal models, and did

not touch the problem of off-target. As a critical evaluation criteria of genome editing

tool, off-target of CRISPR/Cpf1 system remains to be further studied.

\section{METHODS}

\section{RNA in vitro transcription.}

AsCpf1 was synthesized by IGE Biotech Ltd. and cloned into the pCS2+ vector.

AsCpf1 and SpCas9 mRNA were transcribed using the mMessage mMachine SP6 Kit

(Thermo Fisher). All crRNAs and sgRNAs were synthesized using the TranscriptAid 
Verified the effectiveness of AsCpf1 in a variety of species

T7 High Yield Transcription Kit (Thermo Fisher). ssDNA oligos (Supplemental Material)

corresponding to the reverse complement of the target sequence were synthesized

from Thermo Fisher Scientific Inc and IDT, and annealed to a short T7 primer. All

RNAs were purified using the ZR-96 RNA Clean Kit (Zymo).

mESCs culture and transfection.

Mouse embryonic stem cells (mESCs) were maintained on feeder layers or feeder

free with mESC-2i medium (DMEM, 15\%FBS, NEAA, GlutaMAX, PD0325901,

Chir99021, LIF) at $37^{\circ} \mathrm{C}$ with $5 \% \quad \mathrm{CO}_{2}$. Plasmids were transfected by

Mouse ES Cell Nucleofector kit (Lonza) following the manufacturer's instruction.

RNAs were transfected by Lipofectamine RNAiMAX (Thermo Fisher) following the manufacturer's instruction, and riboTRACER ${ }^{\mathrm{TM}}$ Green (Ribobio) as the positive control.

HEK293T cells culture and transfection.

HEK293T cell lines were maintained in Dulbecco's modified Eagle's medium (DMEM)

supplemented with $10 \% \mathrm{FBS}$ ( $\mathrm{HyClone}$ ) at $37^{\circ} \mathrm{C}$ with $5 \% \mathrm{CO}_{2}$ incubation. Plasmids

were transfected by Lipofectamine 3000 (Thermo Fisher) following the manufacturer's 
Verified the effectiveness of AsCpf1 in a variety of species

instruction. RNAs were transfected by Lipofectamine RNAiMAX (Thermo Fisher)

following the manufacturer's instruction.

Mouse embryos acquisition, RNAs microinjection, and culture.

All animal experiments and Protocols were approved by the Institutional Animal Care

and Use Committees (IACUC) of the Laboratory Animal Research Center at

Guangzhou Institutes of Biomedicine and Health (Permit Number:2015009). C57BL/6

(B6) mouse strains were used as embryo donors. Female B6 mice (6-8 weeks old)

were super-ovulated by intraperitoneal injections of 5 IU pregnant mare serum

gonadotropin (PMSG, Ningbo Renjian Pharmaceutical Co., Ltd, Ningbo, China) and 5

IU human chorionic gonadotropin (hCG, Ningbo Renjian Pharmaceutical Co., Ltd,

Ningbo, China) at 48-hour intervals. The fertilized embryos were collected from

oviducts of super-ovulated female after mating with B6 stud males. RNAs (100ng/uL

Cpf1/Cas9 mRNA and 50ng/uL crRNA/sgRNA) was injected into the cytoplasm of

fertilized eggs with well recognized pronuclei in M2 medium (Millipore). The injected

zygotes were cultured in $\mathrm{G} 1 / \mathrm{G} 2$ medium (Vitrolife) at $37^{\circ} \mathrm{C}$ under $6 \% \mathrm{CO}_{2}$ in air until

blastocyst stage by 3.5 days. 
Verified the effectiveness of AsCpf1 in a variety of species

\section{Pig oocyte/embryos acquisition, activation, RNAs microinjection, and culture.}

Briefly, pig ovaries were collected from a local slaughterhouse and transported to the laboratory in $0.9 \%$ saline with penicillin and streptomycin at $35^{\circ} \mathrm{C}$ to $39^{\circ} \mathrm{C}$. Cumulus oocyte complexes(COCs) were aspirated from antral follicles using $10 \mathrm{ml}$ syringe.

After washed 3 times using maturation medium, Tissue Culture Medium 199 (Gibco), which was supplemented with $0.1 \%(\mathrm{w} / \mathrm{v})$ polyvinyl alcohol (Sigma), $3.05 \mathrm{mM}$ D-glucose, $0.91 \mathrm{mM}$ sodium pyruvate (Sigma), $0.57 \mathrm{mM}$ cysteine (Sigma), $0.5 \mathrm{mg} / \mathrm{mL}$ luteinizing hormone (LH Sigma), $0.5 \mathrm{mg} / \mathrm{mL}$ follicle stimulating hormone (FSH Sigma), $10 \mathrm{ng} / \mathrm{mL}$ epidermal growth factor (Sigma), 10\% (v/v) porcine follicular fluid, $75 \mathrm{mg} / \mathrm{mL}$ penicillin $\mathrm{G}$, and $50 \mathrm{mg} / \mathrm{mL}$ streptomycin, the COCs were transferred to maturation medium for $42-44 \mathrm{~h}$. Then, the matured oocytes were released from the cumulus cells by vigorous vortexing for $5 \mathrm{~min}$ in TL-HEPES containing $0.1 \%$ hyaluronidase (Sigma) and actived in activation medium $\left(0.3 \mathrm{M}\right.$ mannitol, $1 \mathrm{mM} \mathrm{CaCl} \mathrm{Cl}_{2} \cdot 2 \mathrm{H}_{2} \mathrm{O}, 0.1 \mathrm{mM}$ $\mathrm{MgCl}_{2} \cdot 6 \mathrm{H}_{2} \mathrm{O}$, and $0.5 \mathrm{mM}$ HEPES) with 2 successive DC pulses of $120 \mathrm{~V} / \mathrm{mm}$ for 30 $\mu$ seconds using an electrofusion instrument (CF-150B, BLS, Hungary). After recovered for 30 mins, the active oocytes were injected with 200ng/uL Cpf1/Cas9 
Verified the effectiveness of AsCpf1 in a variety of species

mRNA and 20ng/uL crRNA/sgRNA and $\mathrm{ddH}_{2} \mathrm{O}$ as control. The injected embryos were cultured in PZM3 for 6 days and the blastocysts were collected for analysis.

\section{Zebrafish embryos microinjection and culture.}

Wild type Tu line embryos were raised at $28.5^{\circ} \mathrm{C}$. One-cell stage zebrafish embryos

were injected with $2 \mathrm{~nL}$ of a solution containing $200 \mathrm{ng} / \mathrm{\mu L}$ Cpf1/Cas9 mRNA/protein and $20 \mathrm{ng} / \mu \mathrm{L} \mathrm{crRNA} / \mathrm{sgRNA}$. For exogenous targets test, injection solution was included $10 \mathrm{pg} / \mathrm{uL}$ pTg-mTet2 plasmid. The injected embryos were cultured in 1x E3 mediumv (5.0 mM NaCl, $\left.0.17 \mathrm{mM} \mathrm{KCl,} 0.33 \mathrm{mM} \mathrm{CaCl}, 0.33 \mathrm{mM} \mathrm{MgSO}_{4}, \mathrm{pH} 7.4\right)$ for 2 days, and then were collected for analysis.

\section{Xenopus embryos microinjection and culture.}

One-cell stage xenopus embryos were injected with $2 \mathrm{~nL}$ of a solution containing

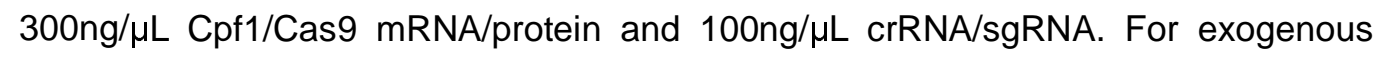
targets test, injection solution was included $10 \mathrm{pg} / \mathrm{uL} \mathrm{pTg-mTet2} \mathrm{plasmid.} \mathrm{The} \mathrm{injected}$ embryos were cultured in 0.1x MBS medium (1x MAS: $88 \mathrm{mM} \mathrm{NaCl}, 2.4 \mathrm{mM} \mathrm{NaHCO}_{3}$, $1 \mathrm{mM} \mathrm{KCl}, 0.82 \mathrm{mM} \mathrm{MgSO}_{4}, 0.33 \mathrm{mM} \mathrm{Ca}\left(\mathrm{NO}_{3}\right)_{2}, 0.41 \mathrm{mM} \mathrm{CaCl}, 10 \mathrm{mM} \mathrm{HEPES} \mathrm{pH}$ 7.4) for 24 hours, and then were collected for analysis. 
Verified the effectiveness of AsCpf1 in a variety of species

\section{T7E1 DSB assay.}

Genomic DNA was extracted using the QuickExtract DNA Extraction Solution

(Epicentre) following the manufacturer's instruction. Genomic region surrounding the

target sites for each gene was PCR amplified. Half PCR products were used for T7

Endonuclease I assay: 10uL PCR products were mixed with 2uL NEB buffer 2 and

$\mathrm{ddH} 20$ to a final volume of $20 \mathrm{uL}$, and subjected to a re-annealing process to enable

heteroduplex formation: $95^{\circ} \mathrm{C}$ for $10 \mathrm{~min}, 95^{\circ} \mathrm{C}$ to $85^{\circ} \mathrm{C}$ ramping at $-2^{\circ} \mathrm{C} / \mathrm{s}, 85^{\circ} \mathrm{C}$ to

$25^{\circ} \mathrm{C}$ at $-0.2^{\circ} \mathrm{C} / \mathrm{s}$, and $25^{\circ} \mathrm{C}$ hold for 1 minute. After re-annealing, products were

treated with $\mathrm{T} 7$ Endonuclease I (NEB) at $37^{\circ} \mathrm{C}$ for $30 \mathrm{~min}$, and analyzed on $10 \%$ TBE

poly-acrylamide gels. Gels were stained with SYBR Gold DNA stain (Thermo Fisher)

for 30 minutes and imaged with Tanon 1600 digital gel image analysis system (Tanon).

The remaining PCR products were gel extraction, cloned into PMD18T (Takara) vector,

and sequenced for counting indels.

\section{In Vitro Enzyme Activity Assay.}

Cpf1 (with Flag tag) expression plasmid was transfected into HEK293T cells as

described below. Cytoplasmic lysates from HEK293T cells were prepared with 
Verified the effectiveness of AsCpf1 in a variety of species

NE-PER Nuclear and Cytoplasmic Extraction Reagents (Thermo Fisher)

supplemented with Protease and Phosphatase Inhibitor (Thermo Fisher). Flag tag

protein was purified by FLAG M purification mammalian expression systems

(Sigma-aldrich) following the manufacturer's instruction. The in vitro enzyme activity

assay was carried out as follows: for a 20 uL cleavage reaction, a certain volume of

endonuclease protein was incubated with 2uL 10X FastDigest Buffer (Thermo Fisher),

$0.5 \mathrm{ug}$ in vitro transcribed RNA and 250ng Sall-linearized pTg- mTet2 plasmids. After

incubation, cleavage reactions were stoped by $6 \mathrm{X}$ Reaction Stop Solution $(60 \mathrm{mM}$

EDTA, $20 \mathrm{mM}$ pH 8.0 Tris-HCl, 0.48\% SDS), purified using ZR-96 DNA Clean \&

Concentrator (Zymo Research) and analysed on 10\% TBE poly-acrylamide gels.

\section{Acknowledgements}

This work were supported by National Natural Science Foundation of China

(31421004, 91419310, 31530038, 31471367, 31271554), National Basic Research

Program of China (973 Program of China, 2012CB966802, 2015CB942803),

Guangdong Science and Technology Project (2014B050504008, 2014B050502012,

2014B030301058, 2014A030304010). The Science and Technology Planning Project 
Verified the effectiveness of AsCpf1 in a variety of species

of Guangdong Province (2016A030303046), and Pearl River Science and Technology

Nova Program of Guangzhou (201710010112).

Guo, X., Zhang, T., Hu, Z., Zhang, Y., Shi, Z., Wang, Q., Cui, Y., Wang, F, Zhao, H. and Chen, Y. (2014). Efficient RNA/Cas9-mediated genome editing in Xenopus tropicalis. Development 141, 707-714.

Jiang, Y., Qian, F., Yang, J., Liu, Y., Dong, F., Xu, C., Sun, B., Chen, B., Xu, X., Li, Y., et al. (2017). CRISPR-Cpf1 assisted genome editing of Corynebacterium glutamicum. Nat Commun 8, 15179.

Kim, D., Kim, J., Hur, J. K., Been, K. W., Yoon, S. H. and Kim, J. S. (2016a). Genome-wide analysis reveals specificities of Cpf1 endonucleases in human cells. Nat Biotechnol 34, 863-868.

Kim, Y., Cheong, S. A., Lee, J. G., Lee, S. W., Lee, M. S., Baek, I. J. and Sung, Y. H. (2016b). Generation of knockout mice by Cpf1-mediated gene targeting. Nat Biotechnol 34, 808-810.

Kleinstiver, B. P., Tsai, S. Q., Prew, M. S., Nguyen, N. T., Welch, M. M., Lopez, J. M., McCaw, Z. R., Aryee, M. J. and Joung, J. K. (2016). Genome-wide specificities of CRISPR-Cas Cpf1 nucleases in human cells. Nat Biotechnol 34, 869-874.

Port, F. and Bullock, S. L. (2016). Augmenting CRISPR applications in Drosophila with tRNA-flanked sgRNAs. Nature Methods 13, 852-854.

Qin, W., Liang, F., Feng, Y., Bai, H., Yan, R., Li, S. and Lin, S. (2015). Expansion of CRISPR/Cas9 genome targeting sites in zebrafish by Csy4-based RNA processing. Cell Res 25, 1074-1077.

Tang, X., Lowder, L. G., Zhang, T., Malzahn, A. A., Zheng, X., Voytas, D. F., Zhong, Z., Chen, Y., Ren, Q., Li, Q., et al. (2017). A CRISPR-Cpf1 system for efficient genome editing and transcriptional repression in plants. Nat Plants 3, 17103.

Toth, E., Weinhardt, N., Bencsura, P., Huszar, K., Kulcsar, P. I., Talas, A., Fodor, E. and Welker, E. (2016). Cpf1 nucleases demonstrate robust activity to 
Verified the effectiveness of AsCpf1 in a variety of species

induce DNA modification by exploiting homology directed repair pathways in mammalian cells. Biol Direct 11, 46.

Wang, H., Yang, H., Shivalila, C. S., Dawlaty, M. M., Cheng, A. W., Zhang, F. and Jaenisch, R. (2013). One-step generation of mice carrying mutations in multiple genes by CRISPR/Cas-mediated genome engineering. Cell 153, 910-918.

Zetsche, B., Gootenberg, J. S., Abudayyeh, O. O., Slaymaker, I. M., Makarova, K. S., Essletzbichler, P., Volz, S. E., Joung, J., van der Oost, J., Regev, A., et al. (2015). Cpf1 is a single RNA-guided endonuclease of a class 2 CRISPR-Cas system. Cell 163, 759-771.

Zhou, X., Xin, J., Fan, N., Zou, Q., Huang, J., Ouyang, Z., Zhao, Y., Zhao, B., Liu, Z., Lai, S., et al. (2015). Generation of CRISPR/Cas9-mediated gene-targeted pigs via somatic cell nuclear transfer. Cell Mol Life Sci 72, 1175-1184. 
Verified the effectiveness of AsCpf1 in a variety of species

\section{Figure legends}

Figure DNA cleavage efficiencies of AsCpf1 and SpCas9 targets at mouse Tet2 locus

in mouse embryos(a), at pig MSTN and Sall1 loci in pig embryos (b), (c). (d) Detected

mouse, zebrafish and xenopus exogenous targets by ETD system in HEK293T cells.

(e) DNA cleavage efficiencies of linear pTg plasmids in zebrafish and xenopus embryo lysates mediated by AsCpf1 protein, AsCpf1 DNA cleavages are indicated by orange arrows. DNA cleavage efficiencies of AsCpf1 targets at zebrafish Tyr locus in zebrafish embryos(f), at xenopus Tyr locus in xenopus embryos (g) (suspected results are marked by purple arrow). Representative SNV caused by AsCpf1 in zebrafish (h) and xenopus Embryos (i). (J) AsCpf1 in vitro enzyme activity assay, DNA cleavage efficiencies have been checked at eight time points and there different temperatures. (k) AsCpf1 enzyme activity curve in the range of $25{ }^{\circ} \mathrm{C}$ to $46{ }^{\circ} \mathrm{C}$, reacted in 10 minutes respectively. 
a. $\mathrm{mTet2}$

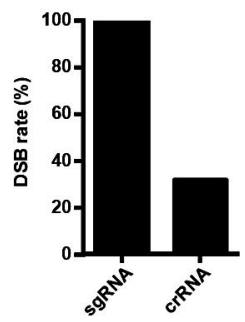

d.
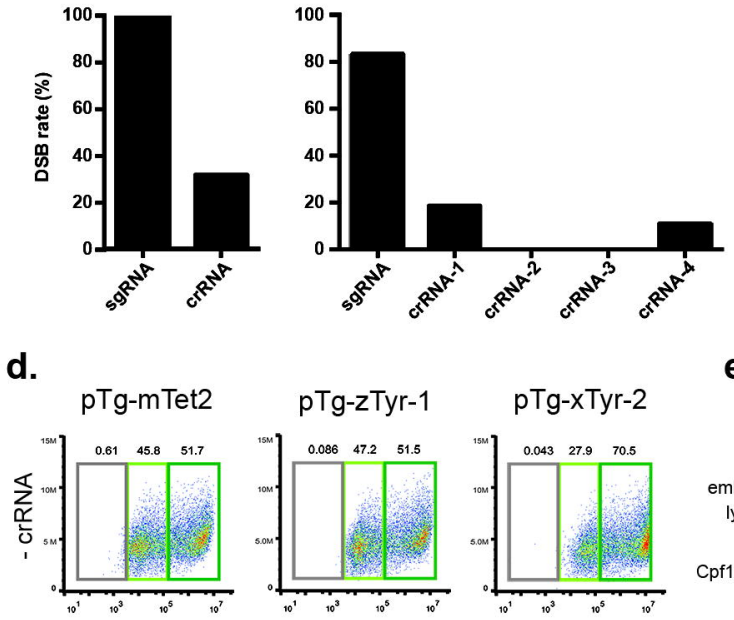

c.

b.

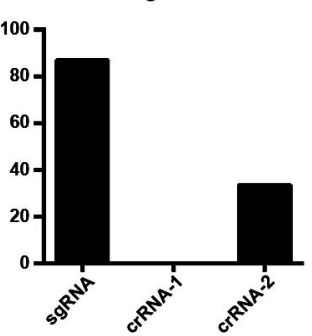

e.

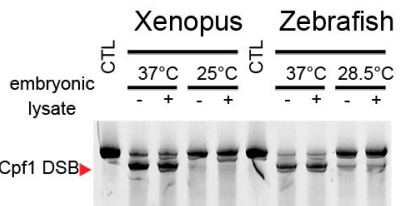

Cpf1 DSB

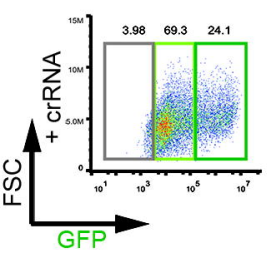

f.

Zebrafish
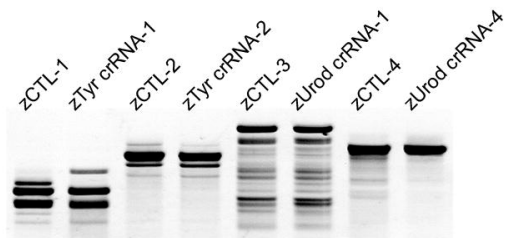

A

h.

zTyr-1

i.

xTyr-2

\section{Xenopus}
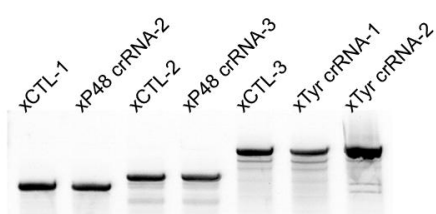

A
5

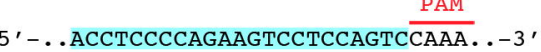

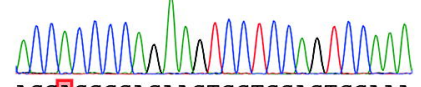

. . ACD国CCCCAGAAGTCCTCCAGTCCAAA. .

j.

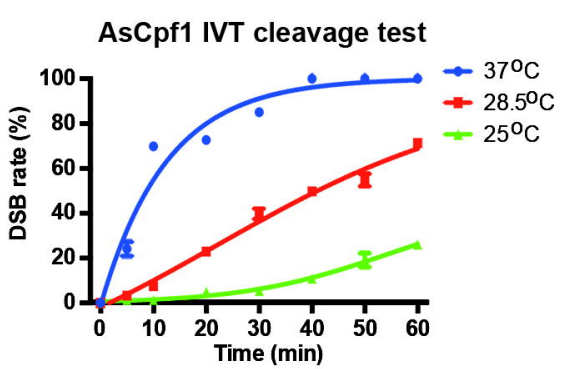

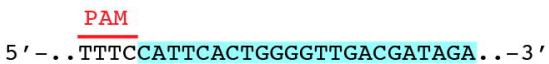
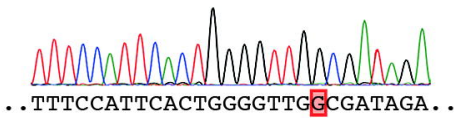

k.

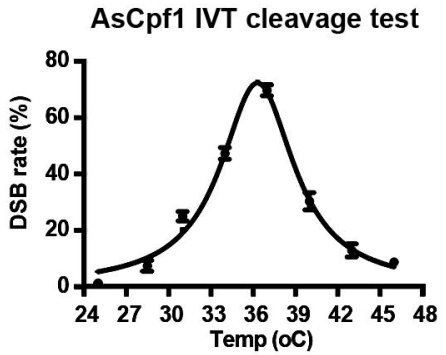

